\title{
Optimal Mother Wavelet Selection for On-line Signature Verification
}

\author{
Xia Xinghua ${ }^{1, a^{*}}$, Luan Fangjun ${ }^{1, b}$ and Yuan Shuai ${ }^{1, c}$ \\ ${ }^{1}$ School of Information and Control Engineering, Shenyang JianZhu University, Shenyang, China

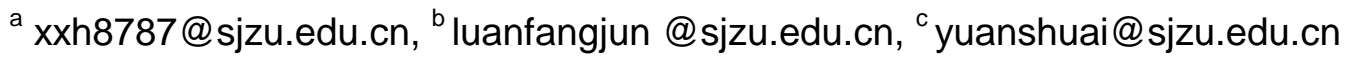

Keywords: On-line signature verification; DTW; WP; Stable spectral feature

\begin{abstract}
On-line signature verification is one of widely acceptable biometrics. Since it can reveal more intrinsic characteristics inherent in writer, spectral information that contains in features can be used to on-line signature verification. In order to extract more effective spectral information, features are decomposed by Wavelet packet (WP) with optimal mother wavelet. Information entropy theory is introduced to reveal the disorder of spectral information contained in signature. The optimal mother wavelet, which is used in WP analysis, is selection based on maximum energy-entropy values to extract more effective information of signature to be used in verification. Because of different writing habits, stabilities of spectral information inherent in signatures maybe varied. To improve the robustness only stable spectral information is extracted. Several experiments are carried out on standard on-line signature dataset MCYT_Subcorpus_100 (DB1), which consists of 5000 signatures from 100 individuals in total. Experiment results demonstrate that the efficiency of on-line signature verification is improved greatly by our proposed method. The best result is given by EER $=3.11 \%$, which also indicates the effectiveness of our proposed methods.
\end{abstract}

\section{Introduction}

On-line signature verification is one of widely acceptable biometrics due to the fact that handwritten signatures have long been established during human daily life. It is difficult to be imitated and forged because signature will be unique and consistency for a given period. On-line signature verification can generally be divided into two groups, i.e. parametric and functional, according to features used in verification [1]. In parametric approach, a set of parameters or vectors are abstracted from sequence of signature, and the signature is represented by series of parameters. When functional approach is concerned, signature is usually characterized in terms of a time function, whose values constitute the feature set. Generally, it is less computation and more efficiency of parametric approach. When functional approach is concerned, it is heavy computation but higher accuracy of on-line signature verification.

Since it can reveal more intrinsic characteristics inherent in writer, spectral information that contains in features can be used to analyze stabilities of on-line signature. Spectral information, which is considered as parametric feature in some cases, is extracted and used in on-line signature verification by mathematical transforms, such as discrete Fourier transform (DFT), discrete Cosine transform (DCT), discrete Wavelet transform (DWT), and Wavelet packet (WP). Rashidi et al. [2] presented a simple and efficient approach based on DCT, which had been applied to 44 time signals. The forward feature selection algorithm was used to search for the best performing feature subsets. Khoh et al. [3] presented a dynamic handwritten signature verification system based on hybrid DWT and DFT. Signature information in a multi-resolution representation was analyzed by DWT, and the feature descriptor of the decomposed sub-bands was extracted by DFT. Yanikoglu et al. [4] used fast DFT to present an on-line signature using a fixed number of coefficients, which led to a fast matching algorithm. Zhang et al. [5] presented a method of on-line signature verification, the zero-across points of features were extracted by DWT. Nanni et al. [6] adopted 1-D DWT to decompose time functional features of signature while the DCT was used to reduce the approximation coefficients vector of a given dimension, and the linear programming descriptor (LPD) classifier was trained using the DCT 
coefficients. Wang et al. [7] proposed a method of on-line signature verification based on WP. The best local features, wavelet bases and sub-bands were selected by conducting several experiments.

Furthermore, in process of DWT, only low-frequency signals are decomposed at each level. To overcome the shortcoming of DWT, WP analysis is used in on-line signature verification for its more powerful ability of describing the details both in low and high frequency. There are different results on the same signal by WP analysis with different mother wavelets. The optimal mother wavelet selection is particularly important. Currently, some methods of mother wavelet selection were mainly based on the statistical analysis of experiment results [7], while other methods of wavelet mother selection were mainly dependent on information entropy theory $[8,9]$.

\section{WP Decomposition of On-line Signature}

Spectral information can be considered as parametric feature, in which can reveal more intrinsic characteristics inherent in writer than functional features. In our works, signatures will be represented in spectral domain by WP decomposition. On-line signatures can be decomposed into two parts by passing through low-pass filter and high pass filter in WP decomposition. Signatures are represented by low-frequency wavelet coefficients and high-frequency wavelet coefficients. Low-frequency wavelet coefficients contain coarse approximation information, called approximation coefficients, or approximation. High-frequency wavelet coefficients contain detail information, called detail coefficients, or details. WP decomposition is given as,

$$
\left\{\begin{array}{c}
d^{j+1,2 i}(k)=\sum_{l} d^{j, i}(l) \cdot \mathrm{h}_{0}^{*}(l-2 k) \\
d^{j+1,2 i+1}(k)=\sum_{l} d^{j, i}(l) \cdot \mathrm{h}_{1}^{*}(l-2 k)
\end{array}\right.
$$

where, $\mathrm{h}_{0}$ and $\mathrm{h}_{1}$ are quadrature mirror filters, ' ${ }^{*}$ ' denotes conjugate. $d^{j, i}$ is the coefficient of WP. $j$ is the level of WP decomposition, and $i$ denotes the number of coefficient sets at level $j, i=0,1, \ldots, 2^{j}-1$. In the WP, both approximation and detail coefficients are decomposed to create the full binary tree.

The signal can also be reconstructed by coefficients,

$$
d^{j, 2 i}(k)=\sum_{l}\left(d^{j+1,2 i}(l) \cdot h_{0}(k-2 l)+d^{j+1,2 i+1}(l) \cdot h_{1}(k-2 l)\right)
$$

As for on-line signature verification, the feature $f_{k}$ is regard as time series signal. The spectral information is obtained by WP decomposition, and time series signal of the feature $f_{k}$ is also obtained using reverse process of WP reconstruction by (2).

\section{Optimal Mother Wavelet Selection}

There are various results on the same signal by WP analysis with different mother wavelets. The optimal mother wavelet selection reasonably is particularly important. In our works, optimal mother wavelet is selected by maximum energy-entropy value. Generally, the greater signal energy, the more amount of information might be contained in the signal. But for a particular system, only effective information would be useful. Entropy is an effective means to evaluate the effectiveness of information contained in signals according to information theory. Smaller entropy value indicates more regular and more effective information contained in signal, vice versa. Thus, more useful information should be extracted with more energy values along with smaller entropy values. Thus, in order to extract more effective and useful information used in on-line signature verification, optimal mother wavelet is selected by maximum energy-entropy value.

During the WP decomposition, energy of signature feature at level $j$ is calculated,

$$
E_{j}=\sum_{i=0}^{2^{j}-1}\left|d^{j, i}\right|^{2}
$$

For all levels, total energy of the feature is obtained,

$$
E_{\text {tol }}=\sum_{j=1}^{L} E_{j}
$$

According to information theory, the entropy of sub-bands at level $\mathrm{j}$ can be given, 


$$
E_{s j}=-\sum_{i=0}^{2^{j}-1} p_{j, i} \cdot \log _{2}\left(p_{j, i}\right)
$$

where, $p_{j, i}$ denotes the energy distribution probability of sub-bands at level j, i.e. $p_{j, i}=\left|d^{j, i}\right|^{2} / E_{j}$. The total entropy of signature feature is obtained,

$$
E_{\text {stol }}=\sum_{j=1}^{L} E_{s j}
$$

The energy-entropy value of on-line signature feature can be defined,

$$
J_{\text {energy-entropy }}=E_{\text {tol }} / E_{\text {stol }}
$$

As for on-line signature verification, the same feature can be WP decomposed with several mother wavelets. The optimal mother wavelet can be selected by maximum energy-entropy value,

$$
\underset{<\psi(t)>}{\arg \max }\left(J_{\text {energy-entropy }}\right)
$$

where, $\left\langle\psi(t)>\right.$ is a set of mother wavelets, $J_{\text {energy-entropy }}$ are energy-entropy values obtained by WP decomposition with different mother wavelets.

With optimal mother wavelet being used in WP decomposition, more effective spectral information can be extracted from signature features.

\section{Verification}

During the process of WP decomposition with optimal mother wavelet, spectral information, which is inherent in signature, is obtained as coefficients. Coefficients of signature, which are regarded as parametric features, would be various because of different habits. Low-frequency coefficients contain basic and coarse approximation information of signatures, while high-frequency coefficients contain detail information of signatures, which is labile to be disturbed. Because of various writing habits, there are different stabilities of coefficients in low- and high- frequency. In order to improve the robustness of on-line signature verification, the labile coefficients of features should be removed.

Because the different writing habits, stable coefficients of given feature would be varied. With these stable coefficients, the stable spectral features are extracted by WP reconstruction. In our works, only low frequency coefficients of feature are selected to be WP reconstruction. Set WP decomposition level $j=3$, there are 8 sets of coefficients, i.e. $\left\{d^{3,0}, d^{3,1}, d^{3,2}, d^{3,3}, d^{3,4}, d^{3,5}, d^{3,6}, d^{3,7}\right\}$, can be obtained. For the robustness of verification, only $\left\{d^{3,0}, d^{3,1}, d^{3,2}, d^{3,3}\right\}$ are selected for WP reconstruction to obtain the stable spectral feature.

As for verificaiton, the dynamic time warping is employed to evaluated the similairy between test signature and reference. For a given individual, let $s(n)=\{(x(n), y(n)), n=1,2, \cdots, N\}$ and $t(m)=\{(x(m), y(m)), m=1,2, \cdots, M\}$ be reference and test signature curves respectively. To obtain the optimal maximum similarity, a distance matrix should be constructed $\mathrm{G}_{N \times M}$, where, elements in row $n$ and column $m$ denotes the distance between $s(n)$ and $t(m)$. The DTW distance can be dynamic programming planned as,

$$
D I S T=\operatorname{Dist}(s(n), t(m))+\min \left\{\begin{array}{c}
d(s(n-1), t(m)) \\
d(s(n-1), t(m-1)) \\
d(s(n), t(m-1))
\end{array}\right.
$$

where, $d(s(n), t(m))=\sqrt{(s(n)-t(m))^{2}}$ is the Euclidean distance between $s(n)$ and $t(m)$.

\section{Experiments}

Several experiments based on large scale dataset of MCYT_Subcorpus_100 (DB1) [10] are carried out. DB1 consists of 5000 on-line signatures from 100 individuals. For each individual, there are 50 
signatures in all, out of these, 25 signatures are genuine and 25 signatures are skilled forgeries. 5 genuine signatures are selected randomly from genuine signatures to be used as references.

10 most used mother wavelets are selected in our experiments of the optimal mother wavelet selection. Experiments are carried out on reference set of DB1. As for database DB1, there are 100 individuals. For given individual, there are 5 references, and 11 original features are extracted for each reference. Thus there are 5500 features in total.

Features are decomposed by WP with 10 mother wavelets at level 3 . The feature number with maximum $J_{\text {energy-entropy }}$ can be counted shown in Table 1 . There are 3698 features can be obtained the maximum $J_{\text {energy-entropy }}$ by WP decomposition with dmey as mother wavelet for DB1. According to statistical results, the most features can be obtained maximum $J_{\text {energy-entropy }}$ by dmey as mother wavelet during WP decomposition. Thus, dmey wavelet is selected as the optimal mother wavelet for WP decomposition and reconstruction in our works.

Table 1 Optimal mother wavelet selection based on maximum energy-entropy value

\begin{tabular}{|c|c|c|}
\hline No. & Mother Wavelets & Numbers of Feature with Max $J_{\text {energy-entropy }}$ \\
\hline 1 & $\mathrm{db} 8$ & 50 \\
\hline 2 & $\mathrm{db} 10$ & 79 \\
\hline 3 & $\mathrm{db} 20$ & 213 \\
\hline 4 & coif4 & 16 \\
\hline 5 & coif5 & 236 \\
\hline 6 & sym5 & 8 \\
\hline 7 & sym8 & 144 \\
\hline $\mathbf{8}$ & dmey & $\mathbf{3 6 9 8}$ \\
\hline 9 & bior2.2 & 46 \\
\hline 10 & bior3.3 & 1009 \\
\hline
\end{tabular}

To illustrate the effectiveness of our proposed method of optimal mother wavelet selection, several mother wavelets are used in WP to extract stable spectral features. Methods of optimal feature sub-space selection and verification are the same as mentioned in this paper. 5 mother wavelets such as dmey, sym8, db20, bior3.3 and coif5 are selected to be used in our experiments. ROC curves of on-line signature verification based on WP analysis with different mother wavelets are shown in Fig. 1. From the ROC curves shown in Fig. 1, it can obtain lowest EER values both on DB1 during WP analysis with demy as mother wavelet. The results illustrate the effectiveness of our proposed method of optimal mother wavelet selection based on maximum energy-entropy value. More effective spectral information can be extracted from signature features by WP analysis with optimal mother wavelet. With the extracted spectral information, we can obtain more effective stable spectral features which can improve the performance of on-line signature verification.

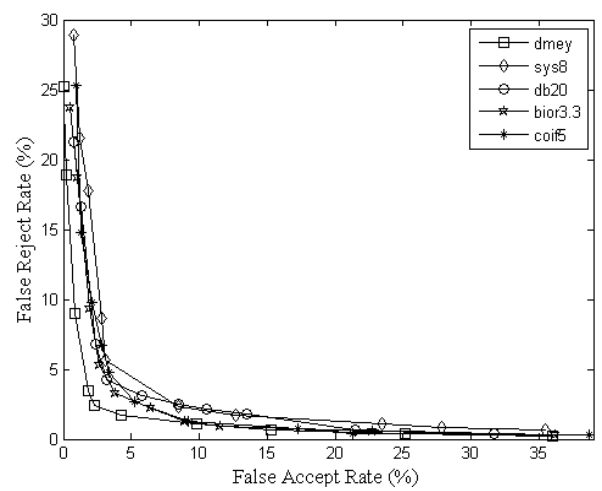

Figure 1. ROC curves of different mother wavelets 
Different coefficients are selected to be used in on-line signature verification to demonstrate the effectiveness of our proposed method. The error rates of verification are shown in Table 2 . Experiment results show that it can obtain the lowest EER with low frequency coefficients $\left\{d^{3,0}, d^{3,1}, d^{3,2}, d^{3,3}\right\}$, which demonstrate the low frequency coefficients can be more stable and robust.

Table 2 Verification performance of different coefficients

\begin{tabular}{|c|c|c|c|}
\hline Coefficients & FRR (\%) & FAR (\%) & EER (\%) \\
\hline$\left\{d^{3,0}, d^{3,3}, d^{3,5}, d^{3,6}\right\}$ & 4.62 & 5.02 & 4.82 \\
\hline$\left\{d^{3,4}, d^{3,5}, d^{3,6}, d^{3,7}\right\}$ & 5.54 & 6.57 & 6.06 \\
\hline$\left\{d^{3,1}, d^{3,3}, d^{3,5}, d^{3,7}\right\}$ & 7.35 & 6.48 & 6.92 \\
\hline$\left\{d^{3,0}, d^{3,2}, d^{3,4}, d^{3,6}\right\}$ & 4.88 & 7.57 & 6.23 \\
\hline$\left\{d^{3,0}, d^{3,1}, d^{3,2}, d^{3,3}\right\}$ & 3.24 & 2.98 & 3.11 \\
\hline
\end{tabular}

\section{Summary}

In this paper, we proposed a method of optimal mother wavelet selection based on max energy-entropy value for on-line signature verification. In order to extract more useful spectral information from signature feature, information entropy theory was introduced in our works. Optimal mother wavelet, which is used in WP analysis, was selected based on max energy-entropy value. Stable spectral information inherent in features was effectively extracted to be used in on-line signature verification. The experiment results demonstrated that effectiveness of on-line signature verification were improved by our proposed method.

\section{References}

[1] Impedovo D, Pirlo G, Automatic Signature Verification: The State of The Art[J], IEEE Transactions on Systems, Man, and Cybernetics, Part C: Applications and Reviews, 2008, 38(5): 609-635.

[2] S. Rashidi, A. Fallah, and F. Towhidkhah, Feature extraction based DCT on dynamic signature verification[J], Scientia Iranica, 2012, 19(6): 1810-1819,.

[3] W. H. Khoh, and T.S. Ong, Score level fusion approach in dynamic signature verification based on hybrid wavelet - Fourier transform[J], Security and Communication Networks, 2014, 7(7): 1067-1078,.

[4] B. Yanikoglu, and A. Kholmatov, Online signature verification using Fourier descriptors[J], EURASIP J. Adv. Signal Process., 2009, 2009:12-12,.

[5] L. Zhang, and Z. Wu, On-line signature verification based on wavelet transform to extract characteristic points[M], Intel. Comput. Signal Process. Pattern Recognit., Berlin, Germany: Springer-Verlag, 2006, 2006:1002-1007.

[6] L. Nanni, and A. Lumini, A novel local on-line signature verification system[J], Pattern Recognit. Lett., 2008, 29(5):559-568,.

[7] K. Wang, Y. Wang, and Z. Zhang, On-line signature verification using wavelet packet[J], Biometrics (IJCB), International Joint Conference on. IEEE, 2011:1-6.

[8] L. Brechet, M. Lucas, and C. Doncarli, Compression of biomedical signals with mother wavelet optimization and best-basis wavelet packet selection[J], IEEE Trans. Biomed. Eng., 2007, 54(12): 2186-2192.

[9] N. Rajpoot, R. Wilson, and F. Meyer, Adaptive wavelet packet basis selection for zerotree image coding[J], IEEE Trans. Image Process., 2003, 12(12):1460-1472.

[10] Ortega-Garcia J, Fierrez-Aguilar J, Simon D. MCYT baseline corpus: a bimodal biometric database [J]. IEE Proceedings-Vision, Image and Signal Processing, 2003, 150(6): 395-401. 